Annales Mathematicae Silesianae 30 (2016), 219-229

DOI: 10.1515/amsil-2015-0013

\title{
A NOTE ON ADDITIVE GROUPS OF SOME SPECIFIC ASSOCIATIVE RINGS
}

\author{
Mateusz Woronowicz
}

\begin{abstract}
Almost complete description of abelian groups $(A,+, 0)$ such that every associative ring $R$ with the additive group $A$ satisfies the condition: every subgroup of $A$ is an ideal of $R$, is given. Some new results for $S R$-groups in the case of associative rings are also achieved. The characterization of abelian torsion-free groups of rank one and their direct sums which are not nil-groups is complemented using only elementary methods.
\end{abstract}

\section{Introduction}

S. Feigelstock studied additive groups of rings whose all subrings are twosided ideals, which resulted in [8]. Such groups are called SI-groups. In [5] we have noted and corrected some inconsistencies in Feigelstock's paper and we have presented new results concerning the structure of $S I$-groups. The aim of the first part of that note is to continue our studies with stronger assumptions. Namely, we investigate abelian groups $A$ such that every associative ring $R$ with the additive group $A$ satisfies the condition: every subgroup of $A$ is an ideal of $R$. Such groups are called $S G I$-groups. We give a full description of torsion $S G I$-groups. We also prove that there does not exist any mixed $S G I$-group. Moreover, we prove that the only up to isomorphism torsionfree $S G I$-group which is not a nil-group, is the group of integers. Similar abelian groups were studied also by A.R. Chekhlov who did not assume the associativity of rings (cf. [6]). Therefore, he obtained his result in a different

Received: 4.03.2015. Revised: 5.10.2015. Accepted: 07.10.2015.

(2010) Mathematics Subject Classification: 20K99.

Key words and phrases: nil-groups, ideals, associative rings. 
way than we did (e.g., his main tool was the tensor product of abelian groups). It turns out that if [7, Conjecture 2.1.4] is true, then the associativity of rings, so important for many algebraists, does not matter for these groups.

$S G I$-groups are related to $S R$-groups studied, for example by A.M. Aghdam, F. Karimi and A. Najafizdeh in [2]. An $S R$-group is an abelian group whose all subgroups are subrings of any (not necessarily associative) ring defined on it. An associative ring $R$ such that any subgroup of its additive group is a subring of $R$ is called an $S$-ring (cf. [14]). This work motivates the concept of the $S$-group understood as an abelian group with the property $S R$ restricted to the class of associative ring. We prove some new results for $S$-groups which allow the conclusion that the properties $S G I$ and $S$ are equivalent. In particular, we prove that the concepts of $S R$-group and $S$-group are equivalent for torsion and mixed groups.

In the second part of the paper, we complement the characterization of abelian torsion-free groups of rank one which are not nil-groups. Since every abelian torsion-free group of rank one can be embedded into the group of rationals, we restrict our considerations to subgroups of the group of rationals. We introduce new simple tools which are useful to prove that a nontrivial subgroup $A$ of the group of rationals is not a nil-group if and only if there exists a ring $R=(A,+, *)$ with a unity. Moreover, we give a simple tool useful to verify whether a direct sum of abelian groups of rank one is a nilgroup (cf. Proposition 4.9 and [7, Corollary 2.1.3]). Our proofs are much more elementary than the proofs previously known.

The topic has long history in algebra. At the same time, it does not lose relevance in modern science. First of all, we note that there are different articles which have been written not only by S. Feigelstock and A. Chekhlov but also by other algebraists lately: Pham Thi Thu Thuy, E. Kompantseva, (cf. [12, 13, 15, 16]).

Symbols $\mathbb{Q}, \mathbb{Z}, \mathbb{P}, \mathbb{N}$ stand for the field of rationals, the ring of integers, the set of all prime numbers, the set of all natural numbers understood as the set of all positive integers, respectively. In this paper, only abelian groups with a traditionally additive notation applied for them, will be considered. If $A$ is an abelian group, then the torsion part of $A$ and the divisible hull of $A$ are denoted by $T(A)$ and $D(A)$, respectively. The set of all ring multiplication on $A$ is denoted by $\operatorname{Mult}(A)$. If $a \in A$, then symbols $o(a)$ and $\langle a\rangle$ stand for the order of $a$ and the cyclic group generated by $a$, respectively. If $\left\{A_{i}: i \in I\right\}$, where $I \neq \emptyset$, is a family of abelian groups and $i \in I$, then $\overline{A_{i}}$ denotes the subgroup of $\bigoplus_{i \in I} A_{i}$ such that the support of an arbitrary element of $\overline{A_{i}}$ is contained in the set $\{i\}$. By $Z(n)$ and $\mathbb{Z}_{n}$ we denote the cyclic group of order $n$ and the ring of integers modulo $n$, respectively. The two-sided ideal $I$ of a ring $R$ is denoted by $I \triangleleft R$. The symbol $R^{+}$stands for the additive group of the ring $R$. If $X \subseteq R$, then the symbol $[X]$ stands for the subring of $R$ generated by $X$. Every abelian group $(A,+, 0)$ can be provided with a ring 
structure in a trivial way by defining $a * b=0$, for all $a, b \in A$. Such a ring is called a null-ring and it is denoted by $A^{0}$. We assume that subrings of $\mathbb{Q}$ possess a unity. The sign function is denoted by sgn. The greatest common divisor of integers $k$ and $l$ is denoted by $\operatorname{GCD}(k, l)$. Throughout the paper the symbol $\cdot$ means the standard multiplication of the field $\mathbb{Q}$.

We remind the reader that an abelian group $A$ is called a $n i l$-group $\left(n i l_{a^{-}}\right.$ group) if on $A$ there does not exist any nonzero (associative) ring multiplication. It follows from [5, Remark 2.6] and [7, Conjecture 2.1.4] that if the concepts of $n i l_{a}$-group and nil-group are not equivalent, then there exists a torsion-free $n i l_{a}$-group of rank more than one which is not a nil-group. The concept connected with nil-groups is a square subgroup which can be understood as follows: given abelian group $A$, the square subgroup $\square A$ of $A$ is the smallest subgroup $B$ of $A$ satisfying the condition: if $R$ is any ring with $R^{+}=A$, then $R^{2} \subseteq B$. If we restrict our consideration to associative rings $R$ with $R^{+}=A$, then we write $\square_{a} A$. More information about square subgroups is available in [1, 3].

The next two section follow partially from results achieved in [5, 8] and they are related to the notion of $S R$-group introduced in [2].

\section{2. $S$-groups}

We remind the reader that an abelian group $A$ is called an $S R$-group if in every (not necessarily associative) ring $R$ with $R^{+}=A$ any subgroup of $A$ is an ideal of $R$. If we restrict our consideration to associative rings, then $A$ is called an $S$-group. This name is associated with $S$-rings that were studied by J.D. O'Neill in [14. Some generalizations of $S$-rings and $S$-groups were also partially investigated by $\mathrm{S}$. Feigelstock in [9] under names $C_{2}$-rings and $S C_{2}$-groups. To the best of our knowledge, issues related to $S R$-groups and $S$-groups have never been researched thoroughly enough, so we give some facts of them below. Moreover, we prove some new results on $S$-groups and $S R$-groups which will be useful to prove that the concepts of the $S$-group and the $S G I$-group are equivalent.

TheOrem 2.1. A torsion abelian group $A$ is an $S$-group if and only if $A_{p}$ is either cyclic or divisible for every prime $p$.

Proof. Compare with [9, Theorem 3.1].

Remark 2.2. Proposition 5.4 of [2] remains true for $S$-groups, i.e., a direct summand of an $S$-group is an $S$-group. 
Observe that [2, Proposition 5.2] can be somewhat generalized.

Proposition 2.3. Let $R$ be a ring with $R^{+}=A$ and let $M$ be a left $R$-module. If $R^{2} \circ M \neq\{0\}$, then $A \oplus M$ is not an $S$-group.

Proof. Since $R^{2} \circ M \neq\{0\}$, there exist $r_{1}, r_{2} \in R$ and $m \in M$ such that $\left(r_{1} r_{2}\right) \circ m \neq 0$. It is easily seen that the function $*:(A \oplus M) \times(A \oplus M) \rightarrow A \oplus M$ given by:

$$
\left.\left(a_{1}, m_{1}\right) *\left(a_{2}, m_{2}\right)=\left(0,\left(a_{1} a_{2}\right) \circ m\right)\right)
$$

is a ring multiplication. Moreover, $(0, x) *(a, y)=(a, x) \circ(0, y)=(0,0)$, for all $a \in A, x, y \in M$, so $P=(A \oplus M,+, *, 0)$ is an associative ring. Define $T=A \oplus\{0\}$. Then $T$ is a subgroup of $P^{+},\left(r_{1}, 0\right),\left(r_{2}, 0\right) \in T$ and $\left.\left(r_{1}, 0\right) *\left(r_{2}, 0\right)=\left(0,\left(r_{1} r_{2}\right) \circ m\right)\right) \notin T$. Hence $T$ is not a subring of $P$ and consequently $A \oplus M$ is not an $S$-group.

COROLlary 2.4. For every nontrivial abelian group $A$, the group $\mathbb{Z}^{+} \oplus A$ is not an $S$-group. Moreover, if $m \leq n$ are positive integer, then $Z\left(p^{n}\right) \oplus Z\left(p^{m}\right)$ is not an $S$-group (that follows also from Theorem 2.1).

THEOREM 2.5. An abelian torsion-free group $A$ is an $S$-group if and only if either $A \cong \mathbb{Z}^{+}$or $A$ is a nil $a_{\text {-group. }}$.

Proof. Suppose that $A$ is a torsion-free $S$-group which is not a $n i l_{a^{-}}$ group. Let $R$ be an associative ring such that $R^{+}=A$ and $R^{2} \neq\{0\}$. It follows from [14, Lemma 2.5] that there exists an ideal $I$ of $R$ such that $I^{2}=\{0\}$ and $R^{+}=I^{+} \oplus\langle x\rangle$, for some $x \in R$ such that $x^{2} \neq 0$. Thus $A \cong I^{+} \oplus \mathbb{Z}^{+}$. Hence, by Corollary 2.4 we get $I=\{0\}$. Therefore $A \cong \mathbb{Z}^{+}$. The opposite implication is obvious.

REMARK 2.6. Notice that the above theorem is the partial answer to the question posed at the end of [2]. The answer is only partial, because we restrict our consideration to the class of associative rings.

Lemma 2.7. Let $p$ and $n$ be a prime and a positive integer, respectively. If $A$ is a non-torsion abelian group, then $G=\mathbb{Z}_{p^{n}}^{+} \oplus A$ is not an $S$-group.

Proof. Define $R=\mathbb{Z}_{p^{n}} \oplus A^{0}$. Take any $a \in A$ such that $o(a)=\infty$. Then $(1, a)^{2}=(1,0)$. Suppose, contrary to our claim, that $(1,0)=k(1, a)$, for some $k \in \mathbb{Z}$. Then $k 1=1$ and $k a=0$. As $o(a)=\infty$ we have $k=0$. Hence $0=1$ in the ring $\mathbb{Z}_{p^{n}}$, which is a contradiction. Thus, $\langle(1, a)\rangle$ is not a subring of $R$ and consequently $G$ is not an $S$-group. 
The next lemma is a consequence of the construction of a ring multiplication given in the proof of [8, Theorem 10]. We present a complete proof for the transparency of the paper.

Lemma 2.8. Let $p$ be a prime and let $A$ be a non-torsion abelian group. Then $G=Z\left(p^{\infty}\right) \oplus A$ is not an $S$-group.

Proof. Take any $x \in Z\left(p^{\infty}\right)$ and $a \in A$ such that $o(x)=p^{2}$ and $o(a)=$ $\infty$. Obviously, the function $\psi:\langle a\rangle \times\langle a\rangle \rightarrow Z\left(p^{\infty}\right)$ defined by the formula $\psi(k a, l a)=(k l) x$, for all $k, l \in \mathbb{Z}$, is bilinear. Therefore [10, Theorem 59.1] implies the existence of a unique homomorphism $\varphi:\langle a\rangle \otimes\langle a\rangle \rightarrow Z\left(p^{\infty}\right)$ satisfying $\psi=\varphi \circ e$. Let $\imath$ be the restriction of $i d_{A \otimes A}$ to $\langle a\rangle \otimes\langle a\rangle$. Then $\imath$ is a monomorphism. Moreover, the group $Z\left(p^{\infty}\right)$ is injective by [10, Theorem 24.5]. Thus there exists a homomorphism $\phi: A \otimes A \rightarrow Z\left(p^{\infty}\right)$ satisfying $\varphi=\phi \circ \imath$. Notice that $\phi(a \otimes a)=(\phi \circ \imath)(a \otimes a)=\varphi(a \otimes a)=(\varphi \circ e)(a, a)=\psi(a, a)=x$. Thus, the multiplication $\left(x_{1}, a_{1}\right) *\left(x_{2}, a_{2}\right)=\left(\phi\left(a_{1} \otimes a_{2}\right), 0\right)$ for all $x_{1}, x_{2} \in Z\left(p^{\infty}\right)$ and $a_{1}, a_{2} \in A$, induces a nontrivial associative ring structure $R$ on $G$. Since $(0, a) *(0, a)=(x, 0) \notin\{0\} \oplus A$, we infer that $R$ is not an $S$-ring. Therefore $G$ is not an $S$-group.

Proposition 2.9. Let $A$ be an $S$-group. Then $A_{p}$ is a direct summand of $A$ for each prime $p$. In particular, if $A$ is an $S$-group then $A_{p}$ is an $S$-group for each prime $p$.

Proof. Take any $p \in \mathbb{P}$. Let $D_{(p)}$ denote the maximal divisible subgroup of $A_{p}$. Then $A=D_{(p)} \oplus H$ for some subgroup $H$ of $A$. Hence $A_{p}=D_{(p)} \oplus H_{p}$. If $H_{p}=\{0\}$, then $A_{p}=D_{(p)}$ and consequently $A_{p}$ is a direct summand of $A$. Now, suppose that $H_{p} \neq\{0\}$. Since $H_{p}$ is a reduced group, it follows from [10, Corollary 27.3] that there exist $n \in \mathbb{N}$ and a subgroup $K$ of $A$ such that $A=D_{(p)} \oplus Z\left(p^{n}\right) \oplus K$. If $K_{p} \neq\{0\}$, then we apply [10, Corollary 27.3] again to infer that $Z\left(p^{n}\right) \oplus Z\left(p^{m}\right)$ is a direct summand of $A$ for some $m \in \mathbb{N}$. Thus, $A$ is not an $S$-group, by Remark 2.2 and Corollary 2.4, which is a contradiction. Therefore $K_{p}=\{0\}$, whence $A_{p}=D_{(p)} \oplus Z\left(p^{n}\right)$ and finally $A_{p}$ is a direct summand of $A$.

The last statement is a direct consequence of the first part of the proof and Remark 2.2.

Theorem 2.10. There does not exist any mixed S-group.

Proof. Suppose, contrary to our claim, that $G$ is a mixed $S$-group. Then, it follows from Proposition 2.9 that there exists $p \in \mathbb{P}$ such that $G_{p} \neq\{0\}$ and $G=G_{p} \oplus H$, for some subgroup $H$ of $G$. Moreover, $G_{p}$ is an $S$-group. Hence, by Theorem 2.1 we need only consider two cases: 
(i) $G_{p}=Z\left(p^{n}\right)$, for some $n \in \mathbb{N}$. Then $G$ is not an $S$-group, by Lemma 2.7 , which is a contradiction.

(ii) $G_{p}$ is divisible. Then $Z\left(p^{\infty}\right) \oplus H$ is a direct summand of $G$, which is not an $S$-group, by Lemma 2.8 . Thus $G$ is not an $S$-group, by Remark 2.2 , which is a contradiction.

Corollary 2.11. Since every SR-group is an S-group, we infer that there does not exist any mixed SR-group. Combining this with Theorem 2.5 and Corollary 3.4 we conclude that the concepts of SR-group and S-group are equivalent exactly if [7, Conjecture 2.1.4] holds.

REMARK 2.12. Since Theorem 2.10 remains true for $S C_{2}$-groups, we infer that in [9, Theorem 3.4] we can assume that $n>2$.

\section{3. $S G I$-groups}

Definition 3.1. An abelian group $A$ is called an $S G I$-group if every associative ring $R$ with $R^{+}=A$ has the property that every subgroup of $A$ is an ideal of $R$.

REMARK 3.2. In [5] we have introduced a new necessary terminology to describe $S I$-groups. We remind the reader that an abelian group $A$ is called

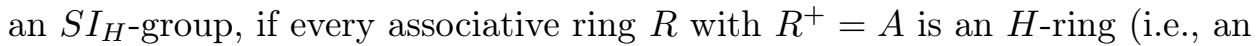
associative ring in which all subrings are two-sided ideals). Since we do not assume that rings are unital, ideals of them are subrings. Hence the property $S$, in conjunction with property $S I_{H}$ gives precisely the property $S G I$. For preliminary knowledge on $S I_{H}$-groups we refer the reader to [5, 8].

Remark 3.2 , Theorem 2.1 and [8, Theorem 7] imply at once the following.

TheOREm 3.3. An abelian p-group $A$ is an SGI-group if and only if either $A$ is divisible or $A=Z\left(p^{n}\right)$ for some positive integer $n$. Moreover, a torsion abelian group $A$ is an $S G I$-group if and only if all its p-primary components $A_{p}$ are $S G I$-groups.

COROLLARY 3.4. For any torsion abelian group A the following conditions are equivalent:

(i) $A$ is an $S R$-group;

(ii) $A$ is an $S$-group;

(iii) $A$ is an $S G I$-group. 
Corollary 3.5. It follows from Remark 3.2 and Theorem 2.5 that an abelian torsion-free group is an SGI-group if and only if it is an S-group. The classification of torsion-free SGI-groups follows also from [5, Theorem $3.10]$.

Corollary 3.6. Theorem 2.10 and Remark 3.2 imply at once that there does not exist any mixed SGI-group.

Corollaries $3.4,3.5$ and 3.6 give at once the following.

THEOREM 3.7. An abelian group is an SGI-group if and only if it is an $S$-group.

\section{Nil-subgroups of the group of rationals}

A main tool which is used to describe the subgroups of $\mathbb{Q}^{+}$is the theory of types (cf. [11, Chapter 85]). We introduce new simple tools which are useful to verify whether a subgroup $A$ of $\mathbb{Q}^{+}$is a nil-group or $A=\square A$. We will use only elementary number theory and very straightforward algebra.

REMARK 4.1. It is a well-known fact that the equation $a x+b y=c$, where $a, b, c \in \mathbb{Z}$, has integer solutions if and only if $\operatorname{GCD}(a, b) \mid c$. That simple fact implies the following useful propositions.

(i) Let $\{0\} \neq A \leq \mathbb{Q}^{+}$and let $m=\min A \cap \mathbb{N}$. If $k \in \mathbb{Z}$ and $n \in \mathbb{N}$ satisfy $k \neq 0, \operatorname{GCD}(k, n)=1$ and $\frac{k}{n} \in A$, then $\frac{m}{n} \in A$. Moreover, $m \mid k$.

(ii) Let $A \leq \mathbb{Q}^{+}$, let $p_{1}<p_{2}<\ldots<p_{s}$ be primes and let $\alpha_{1}, \alpha_{2}, \ldots, \alpha_{s} \in \mathbb{N}$. If $k \in \mathbb{Z}$ and $\operatorname{GCD}\left(k, p_{i}\right)=1$ for each $i=1,2, \ldots, s$, then $\frac{k}{\prod_{i=1}^{s} p_{i}^{\alpha_{i}}} \in A$ if and only if $\frac{k}{p_{i}^{\alpha_{i}}} \in A$, for each $i=1,2, \ldots, s$.

(iii) If $\emptyset \neq P \subseteq \mathbb{P}$, then $\sum_{p \in P}\left[\frac{1}{p}\right]^{+}=\left[\frac{1}{p}: p \in P\right]^{+}$.

Remark 4.2. If $\{0\} \neq A \leq \mathbb{Q}^{+}, m=\min A \cap \mathbb{N}$ and $B=\frac{1}{m} \cdot A$, then $B \leq \mathbb{Q}^{+}, B \cong A$ and $1 \in B$. If $*$ is any nonzero ring multiplication on $B$, then $q_{1} * q_{2} \neq 0$ for some $q_{1}, q_{2} \in B$. Let $k_{i} \in \mathbb{Z} \backslash\{0\}, n_{i} \in \mathbb{N}$ satisfy $\operatorname{GCD}\left(k_{i}, n_{i}\right)=1$ and $q_{i}=\frac{k_{i}}{n_{i}}$ for $i=1,2$, and let $q=1 * 1$. Then $\left(k_{1} k_{2}\right) q=$ $\left(k_{1} k_{2}\right)(1 * 1)=\left(k_{1} * k_{2}\right)=\left(n_{1} n_{2}\right)\left(q_{1} * q_{2}\right) \neq 0$ so $q \neq 0$. If $a, c \in \mathbb{Z}$ and $b, d \in \mathbb{N}$, then $(b d)\left(\frac{1}{b} * \frac{1}{d}\right)=q$, whence $\frac{1}{b} * \frac{1}{d}=\frac{1}{b} \cdot q \cdot \frac{1}{d}$ and finally $\frac{a}{b} * \frac{c}{d}=\frac{a}{b} \cdot q \cdot \frac{c}{d}$. Therefore $(B,+, *)$ is a subring of the field $(\mathbb{Q},+, \circledast)$ with $x \circledast y=x \cdot q \cdot y$ (which is isomorphic to the field $\mathbb{Q}$ ). Thus, every ring multiplication on $A$ is commutative and associative. 
Definition 4.3. Let $\{0\} \neq A \leq \mathbb{Q}^{+}$and let $m=\min A \cap \mathbb{N}$. The set of all primes $p$ such that $\frac{m}{p} \in A$ is denoted by $\Omega_{A}$. Define $\Omega_{A}^{\infty}=\left\{p \in \Omega_{A}: A=p A\right\}$ and $\Omega_{A}^{0}=\left\{p \in \Omega_{A}: A \neq p A\right\}$.

Example. If $A=\left\langle\frac{7}{18}\right\rangle+\left[\frac{1}{20}\right]^{+}$, then $\Omega_{A}=\{2,3,5\}, \Omega_{A}^{\infty}=\{2,5\}$ and $\Omega_{A}^{0}=\{3\}$.

Remark 4.4. If $p \in \Omega_{A}^{0}$, then (i) and (ii) of Remark 4.1 imply that there exists the maximal $\alpha \in \mathbb{N}$ such that $\frac{m}{p^{\alpha}} \in A$.

Remark 4.5 ([4, Lemma 2.5]). Let $\{0\} \neq A \leq \mathbb{Q}^{+}$and let $p \in \mathbb{P}$. Then $p \in \Omega_{A}^{\infty}$ if and only if $m\left[\frac{1}{p}\right]^{+} \leq A$, where $m=\min A \cap \mathbb{N}$.

Directly from Remarks $4.1,4.4$ and 4.5 we obtain the following.

Corollary 4.6. Let $A$ be a subgroup of $\mathbb{Q}^{+}$such that $1 \in A,\left|\Omega_{A}^{0}\right|<\infty$, $\Omega_{A}^{0} \neq \emptyset$ and $\Omega_{A}^{\infty} \neq \emptyset$. Let $n=\prod_{p \in \Omega_{A}^{0}} p^{\alpha_{p}}$, where $\alpha_{p}$ is the maximal natural number such that $\frac{1}{p^{\alpha_{p}}} \in A$ for each $p \in \Omega_{A}^{0}$. Then $A=\left\langle\frac{1}{n}\right\rangle+\left[\frac{1}{p}: p \in \Omega_{A}^{\infty}\right]^{+}$.

Proposition 4.7 (44, Proposition 3.3]). If $A$ is a nontrivial subgroup of $\mathbb{Q}^{+}$such that $\left|\Omega_{A}^{0}\right|=\infty$, then $A$ is a nil-group.

The next result shows that the description of non-nil-subgroups of $\mathbb{Q}^{+}$can be obtained in an elementary way, without using types and the tensor product of abelian groups.

THEOREM 4.8. Let $A$ be a nontrivial subgroup of $\mathbb{Q}^{+}$. The following conditions are equivalent:

(i) $A$ is not a nil-group;

(ii) $\left|\Omega_{A}^{0}\right|<\infty$;

(iii) $A \cong\langle x\rangle+S^{+}$, where $x \in \mathbb{Q}^{+}$and $S$ is some subring of the field $\mathbb{Q}$;

(iv) there exists a ring $R$ with a unity such that $R^{+}=A$;

(v) $\square A=\square_{a} A=A$;

(vi) $t(A)^{2}=t(A)$, where $t(A)$ denotes the type of $A$ (cf. [11, Chapter 85);

(vii) there exists a ring $R$ with $R^{+}=A$ such that $R \cong n\left[p_{i}^{-1}: i \in I\right]$, for some $n \in \mathbb{N}$ and $\left\{p_{i}: i \in I\right\} \subseteq \mathbb{P}$ such that $G C D\left(n, p_{i}\right)=1$, for all $i \in I$;

(viii) if $B=\frac{1}{m} \cdot A$, where $m=\min A \cap \mathbb{N}$, then $n(B \cdot B) \subseteq B$ for some $n \in \mathbb{N}$.

Proof. Implications (i) $\Rightarrow$ (ii) and (vi) $\Rightarrow$ (vii) follows directly from Proposition 4.7 and [11, Theorem 121.1], respectively. Theorem 121.1 of [11] implies also (v) $\Rightarrow$ (vi), because from $\square A=A$ and $A \neq\{0\}$ it follows that $A$ 
is not a nil-group. If (ii) holds, the first part of Remark 4.2 and Corollary 4.6 imply that $A \cong\left\langle\frac{1}{n}\right\rangle+\left[\frac{1}{p}: p \in \Omega_{A}^{\infty}\right]^{+}$for some $n \in \mathbb{N}$. Moreover, $x * y=x \cdot n \cdot y$ is a ring multiplication on $\left\langle\frac{1}{n}\right\rangle+\left[\frac{1}{p}: p \in \Omega_{A}^{\infty}\right]^{+}$and $\frac{1}{n}$ is a unity of that multiplication. This completes proofs of (ii) $\Rightarrow$ (iii) and (iii) $\Rightarrow$ (iv). If $R$ is a ring with a unity such that $R^{+}=A$, then $R^{2}=R$ so $\square A=A$. Moreover, every torsion-free ring of rank one is associative, so $\square_{a} A=\square A$. This completes the proof of (iv) $\Rightarrow(\mathrm{v})$. If (vii) holds, then $A$ is not a nil-group, by [11, Theorem 121.1], so $B$ also is not a nil-group by the first part of Remark 4.2 . Hence by already proved implication (i) $\Rightarrow$ (ii) we infer (as above) that there exists $n \in \mathbb{N}$ such that $x * y=x \cdot n \cdot y$ is a ring multiplication on $B$. Hence $n(B \cdot B)=B * B \subseteq B$. This completes the proof of (vii) $\Rightarrow$ (viii). To prove the implication (viii) $\Rightarrow$ (i) suppose that $A$ is a nil-group. Then $B$ is also nil-group so $\left|\Omega_{B}^{0}\right|=\infty$. Let $r \in \mathbb{N}$. Take any $p \in \Omega_{B}^{0}$ such that $p \nmid r$. If $\alpha=\max \left\{s \in \mathbb{N}: \frac{1}{p^{s}} \in B\right\}$, then $r \frac{1}{p^{2 \alpha}} \in(r(B \cdot B)) \backslash B$.

Proposition 4.9. Let $I \neq \emptyset$, let $A_{i}$ be a subgroup of $\mathbb{Q}^{+}$such that $1 \in A_{i}$, for each $i \in I$, and let $A=\bigoplus_{i \in I} A_{i}$. If $\square A \neq\{0\}$, then $n\left(A_{i} \cdot A_{j}\right) \subseteq A_{k}$ for some $i, j, k \in I$ and $n \in \mathbb{N}$. Moreover, if there exist $i, j, k \in I$ and $n \in \mathbb{N}$ such that $n\left(A_{i} \cdot A_{j}\right) \subseteq A_{k}$, then there exists an associative and commutative ring $R$ with $R^{+}=A$, which is not a null-ring. In particular, $\square_{a} A \neq\{0\}$.

Proof. Let $*$ denote an arbitrary nonzero ring multiplication on $A$. Then there exist $i, j, k \in I$ and $a, c \in A$ such that $\pi_{k}\left(\pi_{i}(a) * \pi_{j}(c)\right) \neq 0$, where $\pi_{t}$ denotes the natural projection of $A$ onto $\overline{A_{t}}$, for $t=i, j, k$. It follows from [11, Theorem 119.1] that there exists a ring $R=(D(A),+, \circledast)$ such that $(A,+, *)$ is a subring of $R$. Let $\varphi_{t}$ be the natural injection of $\mathbb{Q}^{+}$into $D(A)$ such that $\varphi_{t}\left(\mathbb{Q}^{+}\right)$is the $t$-th direct summand of $D(A)$, for $t=i, j$, and let $\phi: \overline{A_{k}} \rightarrow A_{k}$ be the natural isomorphism. Define $\vartheta=\phi \circ \pi_{k}$ and $q_{1} \odot q_{2}=\vartheta\left(\varphi_{i}\left(q_{1}\right) \circledast \varphi_{j}\left(q_{2}\right)\right)$ for all $q_{1}, q_{2} \in \mathbb{Q}^{+}$. As $\vartheta, \varphi_{i}, \varphi_{j}$ are additive homomorphisms and $\circledast$ is a nonzero ring multiplication we infer that $\odot$ is a nonzero ring multiplication on $\mathbb{Q}^{+}$. Hence by Remark 4.2 there exist $0 \neq$ $l \in \mathbb{Z}$ and $m \in \mathbb{N}$ such that $q_{1} \odot q_{2}=q_{1} \cdot \frac{l}{m} \cdot q_{2}$ for all $q_{1}, q_{2} \in \mathbb{Q}^{+}$. Let $s=\operatorname{sgn}(l) \cdot m$. Then the ring multiplication $\square$ on the group $\mathbb{Q}^{+}$given by $q_{1} \odot q_{2}=q_{1} \cdot|l| \cdot q_{2}$, for all $q_{1}, q_{2} \in \mathbb{Q}^{+}$, is the $s$-th multiple of $\odot$ in the group $\operatorname{Mult}\left(\mathbb{Q}^{+}\right)$. Hence, for all $x \in A_{i}$ and $y \in A_{j}$ we have $|l|(x \cdot y)=x \sqcup y=$ $s(x \odot y)=s \phi\left(\pi_{k}\left(\pi_{i}\left(\varphi_{i}(x)\right) * \pi_{j}\left(\varphi_{j}(x)\right)\right)\right) \in A_{k}$. Thus it is sufficient to put $n=|l|$.

If $\square A_{i} \neq\{0\}$ for some $i \in I$, then the assertion is obvious. Suppose that $\square A_{i}=\{0\}$ for all $i \in I$. It follows from Theorem 4.8 that we can exclude the case $i=j=k$. Therefore we need only consider three cases: 
(i) $i=j$ and $i \neq k$. Then $\left(a_{1}, c_{1}\right) *\left(a_{2}, c_{2}\right)=\left(0, n\left(a_{1} \cdot a_{2}\right)\right)$ is a nonzero commutative ring multiplication on $A_{i} \oplus A_{k}$. If $R=\left(A_{i} \oplus A_{k},+, *\right)$, then $R *(R * R)=(R * R) * R=\{0\}$ so $R$ is an associative ring. Since the group $A_{i} \oplus A_{k}$ can be embedded into $A$ in a natural way, we are able to construct an associative and commutative ring $S$ with $S^{+}=A$ using the natural projection of $A$ onto $A_{i}$, the multiplication $*$ defined above and the natural injection of $A_{k}$ into $A$. Therefore the assertion follows.

(ii) $i \neq j$ and $k=i$. Then $n\left(A_{i} \cdot A_{j}\right) \subseteq A_{i}$. As $1 \in A_{i}$ we get $A_{j} \subseteq A_{i} \cdot A_{j}$ so $n A_{j} \subseteq n\left(A_{i} \cdot A_{j}\right)$. Hence $n^{2}\left(A_{j} \cdot A_{j}\right)=n\left(\left(n A_{j}\right) \cdot A_{j}\right) \subseteq\left(A_{i} \cdot A_{j}\right) \subseteq A_{i}$ and the assertion follows from $(i)$ of that proof.

(iii) The elements $i, j, k$ are pairwise distinct. A simple computation shows that $\left(a_{1}, b_{1}, c_{1}\right) *\left(a_{2}, b_{2}, c_{2}\right)=\left(0,0, n\left(a_{1} \cdot b_{2}+b_{1} \cdot a_{2}\right)\right)$ is a nonzero associative and commutative ring multiplication on $A_{i} \oplus A_{j} \oplus A_{k}$. Similarly as in $(i)$ we infer that the assertion follows.

Example. If $A=\left\langle\frac{1}{p}: p \in \mathbb{P}\right\rangle$ and $B=\left\langle\frac{1}{p^{2}}: p \in \mathbb{P}\right\rangle$, then $\Omega_{A}^{0}=\Omega_{B}^{0}=\mathbb{P}$ so $\square A=\square B=\{0\}$, by Theorem 4.8. Since $A \cdot A \subseteq B$, Proposition 4.9 implies that $\square_{a}(A \oplus B) \neq\{0\}$.

REMARK 4.10. The ring multiplications constructed in the proof of Proposition 4.9 are useful to proving [2, Theorem 5.9] in an elementary way, without using types of torsion-free groups of rank one.

Acknowledgment. The author is grateful to Professor Ryszard R. Andruszkiewicz who introduced him to this subject. The author expresses his great gratitude to the anonymous Reviewers for their help during the preparation of the paper, especially for their insightful observations.

\section{References}

[1] Aghdam A.M., Square subgroup of an Abelian group, Acta. Sci. Math. 51 (1987), 343348.

[2] Aghdam A.M., Karimi F., Najafizadeh A., On the subgroups of torsion-free groups which are subrings in every ring, Ital. J. Pure Appl. Math. 31 (2013), 63-76.

[3] Aghdam A.M., Najafizadeh A., Square submodule of a module, Mediterr. J. Math. 7 (2010), no. 2, 195-207.

[4] Andruszkiewicz R.R., Woronowicz M., On associative ring multiplication on abelian mixed groups, Comm. Algebra 42 (2014), no. 9, 3760-3767.

[5] Andruszkiewicz R.R., Woronowicz M., On SI-groups, Bull. Aust. Math. Soc. 91 (2015), 92-103.

[6] Chekhlov A.R., On abelian groups, in which all subgroups are ideals, Vestn. Tomsk. Gos. Univ. Mat. Mekh. (2009), no. 3(7), 64-67 
[7] Feigelstock S., Additive groups of rings. Vol. I, Pitman Advanced Publishing Program, Boston, 1983.

[8] Feigelstock S., Additive groups of rings whose subrings are ideals, Bull. Austral. Math. Soc. 55 (1997), 477-481.

[9] Feigelstock S., Rings in which a power of each element is an integral multiple of the element, Archiv der Math. 32 (1979), 101-103.

[10] Fuchs L., Infinite abelian groups. Vol. I, Academic Press, New York-London, 1970.

[11] Fuchs L., Infinite abelian groups. Vol. II, Academic Press, New York-London, 1973.

[12] Kompantseva E.I., Absolute nil-ideals of Abelian groups, Fundam. Prikl. Mat. 17 (2012), no. 8, 63-76.

[13] Kompantseva E.I., Abelian dqt-groups and rings on them, Fundam. Prikl. Mat. 18 (2013), no. 3, 53-67.

[14] O'Neill J.D., Rings whose additive subgroup are subrings, Pacific J. Math. 66 (1976), no. 2, 509-522.

[15] Pham Thi Thu Thuy, Torsion abelian RAI-groups, J. Math. Sci. (N. Y.) 197 (2014), no. $5,658-678$.

[16] Pham Thi Thu Thuy, Torsion abelian afi-groups, J. Math. Sci. (N. Y.) 197 (2014), no. $5,679-683$.

Institute of Mathematics

UNIVERSITY OF BIAEYSTOK

CioŁkowskiego $1 \mathrm{M}$

15-267 BiAŁYSTOK

POLAND

e-mail: mworonowicz@math.uwb.edu.pl 